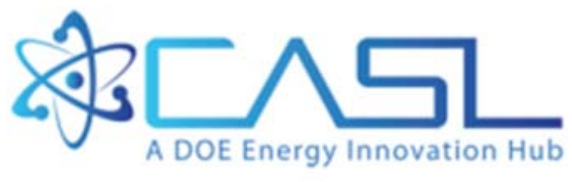

\title{
VERA and VERA-EDU 3.5 Release Notes
}

July 26, 2016
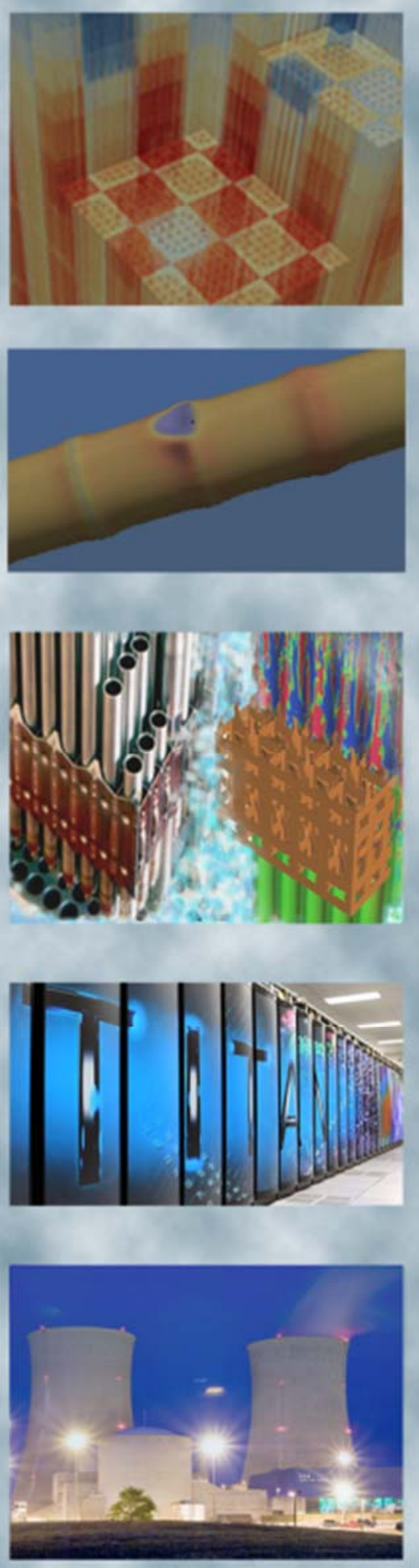


\section{DOCUMENT AVAILABILITY}

Reports produced after January 1, 1996, are generally available free via US Department of Energy (DOE) SciTech Connect.

Website http://www.osti.gov/scitech/

Reports produced before January 1, 1996, may be purchased by members of the public from the following source:

National Technical Information Service

5285 Port Royal Road

Springfield, VA 22161

Telephone 703-605-6000 (1-800-553-6847)

TDD 703-487-4639

Fax 703-605-6900

E-mail info@ntis.gov

Website http://www.ntis.gov/help/ordermethods.aspx

Reports are available to DOE employees, DOE contractors, Energy Technology Data Exchange representatives, and International Nuclear Information System representatives from the following source:

Office of Scientific and Technical Information

PO Box 62

Oak Ridge, TN 37831

Telephone 865-576-8401

Fax 865-576-5728

E-mail reports@osti.gov

Website http://www.osti.gov/contact.html

\begin{abstract}
This report was prepared as an account of work sponsored by an agency of the United States Government. Neither the United States Government nor any agency thereof, nor any of their employees, makes any warranty, express or implied, or assumes any legal liability or responsibility for the accuracy, completeness, or usefulness of any information, apparatus, product, or process disclosed, or represents that its use would not infringe privately owned rights. Reference herein to any specific commercial product, process, or service by trade name, trademark, manufacturer, or otherwise, does not necessarily constitute or imply its endorsement, recommendation, or favoring by the United States Government or any agency thereof. The views and opinions of authors expressed herein do not necessarily state or reflect those of the United States Government or any agency thereof.
\end{abstract}




\section{REVISION LOG}

\begin{tabular}{|c|c|c|l|}
\hline Revision & Date & Affected Pages & \multicolumn{1}{c|}{ Revision Description } \\
\hline 0 & & All & Initial Release \\
\hline & & & \\
\hline & & & \\
\hline & & & \\
\hline
\end{tabular}

\section{Document pages that are:}

Export Controlled ___ None

IP/Proprietary/NDA Controlled None

Sensitive Controlled___ None

\section{Requested Distribution:}

To: Unlimited distribution

Copy: 



\section{GENERAL REMARKS}

The CASL software components provided in this release are under active development, and are subject to change. They have not been fully validated or assessed, and should be used primarily for test, evaluation, and research purposes only.

The Virtual Environment for Reactor Applications components included in this distribution include selected computational tools and supporting infrastructure that solve neutronics, thermal-hydraulics, fuel performance, and coupled neutronics-thermal hydraulics problems. The infrastructure components provide a simplified common user input capability and provide for the physics integration with data transfer and coupled-physics iterative solution algorithms.

Neutronics analysis can be performed for 2D lattices, 2D core and 3D core problems for pressurized water reactor geometries that can be used to calculate criticality and fission rate distributions by pin for input fuel compositions. MPACT uses the Method of Characteristics transport approach for 2D problems [1]. For 3D problems, MPACT uses the 2D/1D method which uses 2D MOC in a radial plane and diffusion or $\mathrm{SP}_{\mathrm{n}}$ in the axial direction. MPACT includes integrated cross section capabilities that provide problem-specific cross sections generated using the subgroup methodology. The code can be executed both 2D and 3D problems in parallel to reduce overall run time.

A thermal-hydraulics capability is provided with CTF (an updated version of COBRA-TF) that allows thermal-hydraulics analyses for single and multiple assemblies using the simplified VERA common input [2]. This distribution also includes coupled neutronics/thermal-hydraulics capabilities to allow calculations using MPACT coupled with CTF.

The VERA fuel rod performance component BISON calculates, on a 2D or 3D basis, fuel rod temperature, fuel rod internal pressure, free gas volume, clad integrity and fuel rod waterside diameter [3]. These capabilities allow simulation of power cycling, fuel conditioning and deconditioning, high burnup performance, power uprate scoping studies, and accident performance. Input/Output capabilities include the VERA Common Input (VERAIn) script which converts the ASCII common input file to the intermediate XML used to drive all of the physics codes in the VERA Core Simulator (VERA-CS). VERA component codes either input the VERA XML format directly, or provide a preprocessor which can convert the XML into native input. VERAView is an interactive graphical interface for the visualization and engineering analyses of output data from VERA. The python-based software is easy to install and intuitive to use, and provides instantaneous 2D and 3D images, 1D plots, and alpha-numeric data from VERA multi-physics simulations.

Testing within CASL has focused primarily on Westinghouse four-loop reactor geometries and conditions with example problems included in the distribution.

\section{SYSTEM REQUIREMENTS}

Linux platforms with functioning gcc, g++ and gfortran compilers and X11 libraries are supported. Systems with 32 cores or greater are recommended. 
Detailed system software and third party library requirements are specified in the provided VERA Installation Guide.

This distribution has been tested and verified to install and execute on the following OS distributions:

- CentOs 6.6

- Ubuntu 14.04.1

- SUSE 11 SP4

- Fedora 21

- CrayOS

The VERAView output viewer tool requires Canopy (https://www.enthought.com/products/canopy/) which itself requires (https://support.enthought.com/hc/en-us/articles/204273874-Canopy-MinimumHardware-Requirements):

- Modern Operating System:

o Windows $7,8.1$, or 10

o Mac OS X 10.9 or higher, 64-bit

o Linux: RHEL 5/6, 64-bit (almost all libraries also work in Ubuntu)

- $\quad$ x86 CPU (Intel / AMD architecture)

- 4 GB RAM

- 5 GB free disk space

\section{INSTALLATION}

Detailed installation instructions are provided in the VERA Installation Guide located in the distribution tarball under the VERA/doc/installation_guide folder and with the documentation delivered with the distribution.

\section{DOCUMENTATION}

The following documentation is made available with the VERA 3.5 release distribution. In addition, all documentation can be viewed or downloaded at www.casl.gov/docs/(document id).pdf or at the URLs indicated below. Other CASL technical reports and publications are available at http://www.casl.gov/journal-and-conference-papers.shtml and http://www.casl.gov/technicalreports.shtml.

Table 1. VERA 3.5 Component Documentation

\begin{tabular}{|l|l|}
\hline Document ID & Document Title \\
\hline CASL-U-2016-1170-000 & VERA 3.5 Installation Guide \\
\hline CASL-U-2014-0014-002 & VERA Common Input User Manual \\
\hline CASL-U-2016-1110-000 & CTF Theory Manual \\
\hline CASL-U-2016-1111-000 & CTF User's Manual \\
\hline CASL-U-2016-1112-000 & CTF Preprocessor User's Manual \\
\hline CASL-U-2016-1113-000 & CTF Validation and Verification \\
\hline CASL-U-2016-1107-000 & MPACT Theory Manual, Version 2.2.0 \\
\hline CASL-U-2016-1108-000 & MPACT Standard Input User's Manual, Version 2.2.0 \\
\hline CASL-U-2016-1109-000 & MPACT VERA Input User's Manual, Version 2.2.0 \\
\hline CASL-U-2014-0038-000 & User Guidelines and Best Practices for CASL VUQ Analysis Using Dakota \\
\hline
\end{tabular}




\begin{tabular}{|l|l|}
\hline CASL-U-2016-1173-000 & DAKOTA6.3 Theory Manual \\
\hline CASL-U-2016-1172-000 & DAKOTA6.3 User's Manual \\
\hline CASL-U-2016-1174-000 & DAKOTA6.3 Reference Manual \\
\hline CASL-U-2016-1175-000 & DAKOTA6.3 Developer's Manual \\
\hline & BISON Theory Manual (https://hison.inl.gov/SiteAssets/BISON Theory version 1 2.pdf) \\
\hline & BISON User's Manual (https://bison.inl.gov/SiteAssets/BISON users version 1 2.pdf) \\
\hline CASL-U-2016-1099-000 & Standalone BISON Through VERA: User's Manual \\
\hline CASL-U-2016-1058-000 & VERAView User's Guide \\
\hline
\end{tabular}

\section{SUPPORT}

Questions, issues, bugs, and suggestions should be reported to support@,casl.gov. Every effort will be made to respond to any requests within a reasonable period of time. Due to active development efforts users may experience some delays.

\section{PHYSICS COMPONENTS INCLUDED IN VERA 3.5}

\subsection{MPACT}

MPACT 2.2.0 is based on the Method of Characteristics transport approach for 2D problems with cross section weighting based on the subgroup methodology [1]. The code can be executed in parallel to reduce overall run time. For 3D problems, MPACT uses the 2D/1D method which uses 2D MOC in a radial plane and diffusion or $\mathrm{SP}_{\mathrm{n}}$ in the axial direction. A 47 group library with subgroup parameters is provided.

New features implemented since MPACT 2.1.0 (VERA 3.4) are:

- Various bug fixes

- More flexible materials input processing

- CMFD Performance improvements.

- MOC Performance Improvements

- Resonance self-shielding performance improvements

- Macroscopic Cross Section calculation performance improvements

- More Simplified T/H models

The following features are considered to be Stable. Footnotes refer to the Known Issues listed in Table 1 of Section 9 below.

- Support for Windows OS (32-bit and 64-bit)

- Support for Linux OS (32-bit and 64-bit)

- Parallel Spatial Decomposition with MPI ${ }^{1}$

- Parallel Angular Decomposition with $\mathrm{MPI}^{2}$

- User defined Macroscopic Cross Sections

- 47-group Macroscopic Cross Section Library Data

- Transport Corrected $\mathrm{P}^{3}$ 
- Export of Mesh to Legacy VTK file for visualization ${ }^{4}$

- 2-D MOC Transport Kernel

- Coarse Mesh Finite Difference (CMFD) Acceleration ${ }^{5}$

- 1-D Nodal Kernels based on NEM-Diffusion and SPn ${ }^{6}$

- 2-D/1-D Full Core Solution ${ }^{5}$

- Multi-State Calculation Capability ${ }^{7}$

- Depletion and Decay

- Critical Boron Search

- Equilibrium Xenon Calculation

- Direct Coupling with COBRA-TF

- Simplified Internal T/H

- General PWR Geometry Modeling

o IFBA

o Control Rods and Control Rod Banks ${ }^{8}$

o Burnable Poison Inserts

o Fission Chamber Detectors

o Grid spacers, Nozzles, Plenum, Baffle, etc.

o Semi-Explicit modeling of grid spacers

- Checkpoint File

- Isotopic Restart File $9,10,11,12$

The following features are considered to be experimental, and are not mature:

- Separate B-10 Depletion of soluble Boron

- Artificially Diffusive CMFD

- Space-Dependent Wielandt Shift or dynamic Wielandt Shifts

- Modeling of BWR lattices

- Simplified CRUD modeling

- Post-Corrector Depletion

- Cycle-to-Cycle Fuel Shuffling (This feature should work without issue in most cases, but it does have known limitations).

o One known issue is that the assm_map in the input must match the shuffle_label card.

o Shuffle by assembly serial number is not supported.

o Changing the axial mesh is not supported for shuffle.

- 3-D MOC Kernels

- Processing of AMPX Working Cross Section Libraries

- 1-D Nodal Kernels based on SANM diffusion

\subsection{CTF}

CTF (an updated version of the COBRA-TF code) is a subchannel thermal-hydraulics code that uses a two-fluid, three-field (i.e. fluid film, fluid drops, and vapor) modeling approach [2]. Both subchannel and three-dimensional (3D) Cartesian forms of nine conservation equations are available for 
LWR modeling. CTF includes a wide range of thermal-hydraulic models important to LWR safety analysis and modeling of normal LWR operating conditions including flow-regime-dependent, twophase wall heat transfer, inter-phase heat transfer and drag, turbulent mixing, void drift, droplet breakup, and quench-front tracking. Due to its 3D capabilities and extensive array of reactor thermal-hydraulic modeling capabilities, CTF has found much use in modeling of LWR rod-bundle transient analysis and Pressurized Water Reactor (PWR) whole-vessel, Loss-Of-Coolant Accident (LOCA) analysis.

Significant changes implemented since VERA 3.4 are:

- Improvement in code parallel performance with addition of new domain refinement option

- Addition of new steady-state stopping criteria based on code solution parameters

- Development of new preprocessor tool for generating BWR models from VERAIn file

- Exposed PETSc numerical controls to input deck

- Addition of outer iteration loop for converging on core pressure distribution in BWR models with assemblies physically separated by channel boxes

- Creation of verification problems and supporting documentation in CTF Validation and Verification Manual

- Addition of new friction correlations that allow the user to specify rod surface roughness

- Addition of feature for DNB analysis that allows the user to perform post-simulation check of DNB rather than during simulation

- Specific flow regime number is now printed to the channel output file

- Expansion of the CTF Coupling Interface in support of coupling CTF/MAMBA to MPACT

- Addition of the Bowring and Groenveld critical heat flux models

- Addition of a feature to track the instantaneous and cumulative true storage and balance of mass and energy

- Addition of developer option build to standalone CTF builds that loosens the test tolerance for non-developers to prevent false test failures due to code portability issues

Stable features:

- Solid modeling capabilities

o Radial conduction

o Nuclear fuel rod models (pellet, gap, and clad regions and UO2 and zircalloy material properties)

- Fluid modeling capabilities

o Solid-to-fluid heat transfer

- single-phase convection

- subcooled/saturated boiling

o Critical heat flux (Departure from Nucleate Boiling)

o Two-phase flow with droplets

o Closure models

- Wall drag and form loss modeling

- Turbulent-mixing and void-drift

- Fluid equation of state

o Incorporation of PETSc solvers 
o Variable size axial meshing

o Grid-heat-transfer enhancement modeling

The following features are available, but not tested with the same rigor as the aforementioned models:

- Solid modeling capabilities

o Fuel rod axial/azimuthal conduction

o Axial mesh refinement (quench front tracking)

o Dynamic gap conductance model (pellet relocation and pellet-clad interaction)

o Zircalloy-water thermal reaction

o Fuel pellet cracking/sintering

- Fluid modeling capabilities

o Non-condensable gas effects

o Post-CHF heat transfer models (are encountered in validation tests, but no validation of models done)

o Droplet entrainment/de-entrainment models (encountered in validation tests, but droplet field is not validated)

o Channel splitting and coalescing

o Channel flow area variations (rod ballooning)

o Grid-directed cross-flow modeling

o Boron-tracking model with consideration of boron precipitation

\subsection{BISON}

VERA includes the capability to predict fuel rod performance utilizing 2D axisymmetric or 3D coupled multi-physics and represents a significant advancement for the modeling/analysis capabilities in LWR fuel rod behavior [3]. The capability is being constructed within the MOOSE/BISON computational framework (from Idaho National Laboratory) that supports:

- 2D and 3D thermomechanics including elasticity, plasticity with strain hardening, creep, large strains, large displacements, and smeared plus explicit cracking;

- Unsteady (transient) conduction heat transfer with time and spatial (axially, radially and potentially azimuthally in a cylindrical fuel element) dependent internal heat generation;

- Gap heat transfer including conduction, radiation and enhanced heat transfer from mechanical contact;

- 2D axisymmetric, generalized plane strain, and plane stress representations, including thermal and mechanical contact interactions between pellets and between the pellet and cladding;

- Mixed dimensional coupling, e.g., combined 2D and 3D numerical representations for coupled global (2D) and local effects (3D) modeling; and

- Utilizes high performance computing platforms to achieve the parallel performance and scalability required to perform coupled multi-physics simulations of full length $3 \mathrm{D}$ representations of the fuel rod components. 
The BISON fuel rod performance code architecture uses the finite element method for geometric representation and a Jacobian-free, Newton-Krylov (JFNK) scheme to solve systems of partial differential equations. The fuel rod performance capability includes models for:

- Clad stress, strain, and strain rate;

- Clad oxidation, hydrogen pickup and hydride formation;

- Pellet stress, strain, and strain rate;

- Fission gas release (transient and pseudo-steady-state);

- Pellet densification and swelling;

- Pellet cracking and relocation;

- Thermal expansion, including pellet hour-glassing;

- Thermal and irradiation creep;

- Thermal conductivity effects due to clad oxidation;

- Material strength and ductility effects due to irradiation;

- Pellet-cladding gap evolution and local stress due to partial contact;

- Pellet stack growth and fuel rod growth;

- Explicit modeling of duplex and triplex clad designs;

The VERA fuel rod performance subcomponent calculates, on a 2D or 3D basis, fuel rod temperature, fuel rod internal pressure, free gas volume, clad integrity and fuel rod waterside diameter. These capabilities allow simulation of power cycling, fuel conditioning and deconditioning, high burnup performance, power uprate scoping studies, and accident performance.

It is important to note that these tools are principally built around the known performance of existing zirconium-based clad with $\mathrm{UO}_{2}$ fuel. Estimates for the global effects of minor modifications to the fuel or clad may be possible; for example, chromia-doped pellets may be simulated with usersupplied models for several of the pellet performance characteristics or steel-based clad may be simulated with similar user-supplied models. Materials such as silicon carbides that do not fit the system paradigm can be simulated but are not likely to provide accurate results.

\subsection{Dakota}

The Dakota 6.3 package [5] manages and analyzes ensembles of simulations to provide broader and deeper perspective for analysts and decision makers. In its simplest mode, Dakota can automate typical parameter variation studies through a generic interface to a physics-based computational model. This can lend efficiency and rigor to manual parameter perturbation studies already being conducted by analysts. Dakota also delivers advanced parametric analysis techniques enabling design exploration, optimization, model calibration, risk analysis, and quantification of margins and uncertainty with such models. It directly supports verification and validation activities. Dakota algorithms enrich complex science and engineering models, enabling an analyst to answer crucial questions of:

- Sensitivity: Which are the most important input factors or parameters entering the simulation, and how do they influence key outputs?

- Uncertainty: What is the uncertainty or variability in simulation output, given uncertainties in input parameters? How safe, reliable, robust, or variable is my system? (Quantification of margins and uncertainty, QMU) 
- Optimization: What parameter values yield the best performing design or operating conditions, given constraints?

- Calibration: What models and/or parameters best match experimental data?

A CASL Technical Report providing user guidelines and best practices for CASL VUQ analysis using Dakota (CASL-U-2014-0038/SANDIA Report SAND2014-2864) is available at: https://dakota.sandia.gov//sites/default/files/documents/SAND-CaslDakotaManual.pdf

The following features are considered to be mature and robust:

- Parameter studies: list, vector, centered, multi-dimensional

- Uncertainty quantification: Monte Carlo and Latin hypercube sampling, local reliability (probability of failure) methods, stochastic expansions (polynomial chaos and stochastic collocation)

- Optimization/calibration: gradient-based local, derivative-free local (pattern search), global (genetic algorithms, direct, etc.), local least squares, surrogate-based local methods

- Surrogate models: polynomials, Gaussian process/Kriging, neural network

- Parameter types: all are mature except discrete string and categorical types

- Interfaces: system, fork, and direct

The following features are considered to be Stable:

- Design and analysis of computer experiments: DDACE grid, random, orthogonal array, OALHS; FSU quasi-Monte Carlo (Halton, Hammersley, centroidal Voronoi tessellation), PSUADE Morris one-at-a-time

- Uncertainty quantification: global reliability (probability of failure) methods, adaptive stochastic expansions, importance sampling (including adaptive, and surrogate-based), Probability of Failure Darts, epistemic interval uncertainty, Dempster-Shafer, Bayesian inference (QUESO, DREAM), incremental LHS

- Optimization: NOMAD directional search, surrogate-based global including EGO, hybrid and pareto optimization

- Surrogate models: MARS, Taylor/TANA, hierarchical and multi-fidelity

- Interfaces: Matlab and Python interfaces; work directory and parallel interface scheduling features refactored recently

The following features are considered to be Experimental or Weakly Tested:

- Design and analysis of computer experiments: DDACE Box-Behnken, central composite designs

- Uncertainty Quantification: D-optimal sampling, Multi-level and multi-fidelity Monte Carlo methods, topology-based adaptive sampling

- Optimization: Genie Opt-Darts, Genie Direct

- Surrogate models: moving least squares, radial basis functions

- Interfaces: Scilab and grid 
- Responses: field data, for both simulations and experiments, including interpolation capability

- Other: active subspace methods for dimension reduction; string/categorical variable support is limited

Release notes for Dakota 6.3 (and previous versions whose capabilities are also included in Dakota 6.3) are available at https://dakota.sandia.gov/content/release-notes. Known Limitations of Dakota 6.3 are listed in Section 9, Table 1, items 14-26.

\section{COUPLED PHYSICS EXECUTABLES}

\subsection{MPACT + CTF}

MPACT has the ability to call CTF to obtain fuel temperatures and moderator density. This is done by directly calling the CTF solver every outer iteration and passing the power distribution. After CTF converges on a given power shape, the temperatures and densities are passed back to MPACT and applied to the cross-sections. A conditional check on the change in temperature and density is performed to determine if the subgroup calculation needs to be rerun to obtain new shielding parameters for the cross-section generation. This procedure continues until MPACT satisfies its internal convergence criteria on eigenvalue and fission source [6-7].

\section{VERA INPUT/OUTPUT TOOLS}

\subsection{VERAIn}

The VERA Common Input (VERAIn) is a PERL script which converts the ASCII common input file to the intermediate XML used to drive all of the physics codes in the VERA Core Simulator (VERACS). VERA component codes either input the VERA XML format directly, or provide a preprocessor which can convert the XML into native input (CTF and BISON).

\subsection{VERAVIew}

VERAView is an interactive graphical interface for the visualization and engineering analyses of output data from VERA. The python-based software is easy to install and intuitive to use, and provides instantaneous 2D and 3D images, 1D plots, and alpha-numeric data from VERA multiphysics simulations. A user's manual is provided giving a brief overview of the software and descriptions of the major features of the application, including examples of each of the encapsulated 'widgets' that have been implemented thus far. VERAView is still under development and large changes in the software are still anticipated.

\section{CAVEATS AND KNOWN ISSUES}

The CASL codes provided in this release are still under active development, and are subject to rapid change. They have not been fully validated or assessed, and should be used for test, evaluation, and research purposes only. 
Users should be aware that not all components in this release may be supported long-term by CASL.

Issues that have been identified during development and testing of this release package are listed in Table 2.

Table 2. Known Issues

\begin{tabular}{|c|c|c|}
\hline Issue ID & Component & Issue \\
\hline 1 & MPACT & $\begin{array}{l}\text { It has been observed that for some MPI distributions the communication routines for the MG MOC } \\
\text { kernels will experience an error in MPI. } \\
\text { * WORKAROUND: Use the shield_moc_kernel } 1 \mathrm{~g} \text { or moc_kernel } 1 \mathrm{~g} \text { option }\end{array}$ \\
\hline 2 & MPACT & $\begin{array}{l}\text { It has been observed that on some platforms (in particular Eos), that depletion cases run with } \\
\text { spatial and angular decomposition will fail. } \\
\text { * WORKAROUND: Use threading instead of angle decomposition. }\end{array}$ \\
\hline 3 & MPACT & $\begin{array}{l}\text { When using TCP0, it has been observed that in some cases that this correction can drive the } \\
\text { solution negative. } \\
\text { * WORKAROUND: Using P2 scattering is recommended at the expense of longer run times. Use } \\
\text { the mOC_kernel mg option. }\end{array}$ \\
\hline 4 & MPACT & $\begin{array}{l}\text { The number of azimuthal divisions in the Flat Source Region mesh of a fuel pin or guide tube in the } \\
\text { visualization file is not representative of the computational mesh. The visualization contains extra } \\
\text { divisions to approximate a curved surface as a series of line segments. }\end{array}$ \\
\hline 5 & MPACT & $\begin{array}{l}\text { For some models it has been observed that the converged solution with and without CMFD are not } \\
\text { in exact agreement. }\end{array}$ \\
\hline 6 & MPACT & $\begin{array}{l}\text { For some 2-D/1-D cases with TCP0, SP3 may have convergence issues. } \\
\text { * WORKAROUND: Use the newHybrid-SP3 nodal kernel and/or a different scattering treatment. }\end{array}$ \\
\hline 7 & MPACT & $\begin{array}{l}\text { Not all values of state variables are carried forward or processed in subsequent states. } \\
\text { * The xenon, tinlet, modden, and t fuel cards are not properly processed for multiple } \\
\text { states. }\end{array}$ \\
\hline 8 & MPACT & $\begin{array}{l}\text { Support for the depletion of absorbing materials in control rods is not yet implemented. The } \\
\text { absorber material in a control rod should be defined in the [CONTROL] block to ensure rod } \\
\text { materials are not depleted. If the materials are placed elsewhere, they may be flagged as } \\
\text { depletable. }\end{array}$ \\
\hline 9 & MPACT & $\begin{array}{l}\text { State variables are not read from the restart file and must be entered in the [STATE] block when } \\
\text { a restart is performed, otherwise default values are used. }\end{array}$ \\
\hline 10 & MPACT & $\begin{array}{l}\text { It has been observed that in cases modeling start-up the restarted solution does not match the } \\
\text { solution of the state where the restart file was written. }\end{array}$ \\
\hline 11 & MPACT & $\begin{array}{l}\text { If the deplete card does not appear in an input with a restart_read then the pin average exposure } \\
\text { edits will not be correct. } \\
\text { * WORKAROUND: If restarting a calculation one can define an additional deplete step to if pin } \\
\text { average exposure edits are desired. }\end{array}$ \\
\hline 12 & MPACT & Changing the mesh is not supported for restarts. \\
\hline 13 & MPACT & $\begin{array}{l}\text { The unit test MPACT libsCMFD_testParCMFD_MPI_4 has been observed to intermittently ( } 4 \% \text { of } \\
\text { time) fail on CentOS } 6.6 \text { virtual machine installations of VERA. This issue has been determined to } \\
\text { be due to a race condition in the test itself, and is not indicative of a code failure. }\end{array}$ \\
\hline 14 & Dakota & Some methods that write intermediate files (e.g. LHS.err) can't be run as concurrent iterators. \\
\hline 15 & Dakota & $\begin{array}{l}\text { dprepro doesn't support the full range of permitted variable descriptors or string variable values. } \\
\text { (Workaround: use variables names currently accepted: Iw, which is [a-zA-Z }] \text { ). }\end{array}$ \\
\hline 16 & Dakota & Support for categorical variables missing from several methods. \\
\hline 17 & Dakota & Importing tabular files into Matlab no longer works straightforwardly due to presence of interface ID. \\
\hline 18 & Dakota & Separate work directories not created for concurrent iterators. \\
\hline 19 & Dakota & D-optimal sampling designs do not work reliably. \\
\hline 20 & Dakota & Coliny COBYLA methods may not return optimal solution. \\
\hline
\end{tabular}




\begin{tabular}{|c|c|c|}
\hline 21 & Dakota & $\begin{array}{l}\text { Sequential hybrid method may only propagate solutions with explicit model specification; use } \\
\text { model_pointer as a workaround. }\end{array}$ \\
\hline 22 & Dakota & Coliny DiRECT method may behave errantly for unbounded optimization domains. \\
\hline 23 & Dakota & Efficient global method may not produce tabular output. \\
\hline 24 & Dakota & PCE methods do not produce statistics when used with design variables; use uncertain instead. \\
\hline 25 & Dakota & User interrupt may not reliably terminate. Dakota when running moga/soga methods. \\
\hline 26 & Dakota & Multi-start method may seg fault when configured with method_name; use method_pointer instead. \\
\hline 23 & $\begin{array}{l}\text { BISON / } \\
\text { MOOSE }\end{array}$ & $\begin{array}{l}\text { The number of processors used by the libmesh build process within MOOSE is controlled by the } \\
\text { environment variables MOOSE_JOBS and LIBMESH_JOBS. If building on less than } 8 \text { cores, it is } \\
\text { advisable to set these environment variables equal to the number of available processors. }\end{array}$ \\
\hline 24 & BISON & $\begin{array}{l}\text { The standalone BISON test suite is experiencing EXODIFF failures. The root cause has been } \\
\text { traced to an issue with test output files missing a t=0 time step point in the exodus file compared to } \\
\text { reference "gold" files. This issue is related to the tests themselves and is not believed to be } \\
\text { reflective of a code failure. }\end{array}$ \\
\hline 25 & CTF & $\begin{array}{l}\text { The channel splitting feature of CTF enables a user to split a large channel into several small } \\
\text { channels in the axial direction or, vice versa, condense several small channels into one large } \\
\text { channel. This can be done only in multi-axial-section models. It has been observed that the code } \\
\text { may create mass in the system when using this feature for transient simulations. }\end{array}$ \\
\hline 26 & CTF & $\begin{array}{l}\text { The dynamic gap conductance model is used to calculate the fuel rod gap conductance as a } \\
\text { function of fill gas temperature and pressure and fuel rod temperature. The alternative model is to } \\
\text { specify a constant gap conductance over time. A known bug exists where the code will overwrite } \\
\text { model data if more than one fuel type is present in the model. However, note that this feature is } \\
\text { more experimental in nature, is minimally tested, and its use is cautioned. }\end{array}$ \\
\hline 27 & CTF & $\begin{array}{l}\text { The grid droplet breakup model is used to divide the droplet field into large and small drops due to } \\
\text { impacting with spacer grids in loss-of-coolant accident conditions. An uninitialized variable has been } \\
\text { detected in this model that leads to unpredictable results. This is an experimental feature and is } \\
\text { minimally tested, so its use is cautioned. }\end{array}$ \\
\hline 28 & CTF & $\begin{array}{l}\text { It has been discovered that the input to the droplet de-entrainment model for the top-quench-front } \\
\text { flow regime (loss-of-coolant accident conditions) is not correct. Modeling droplet flow in accident } \\
\text { conditions is cautioned against until this defect is fixed. }\end{array}$ \\
\hline 29 & CTF & $\begin{array}{l}\text { There is a bug in the way that the calculated critical heat flux is being time-relaxed for cases that } \\
\text { use the W- } 3 \text { correlation. This bug only affects models using the W- } 3 \text { correlation and only transient } \\
\text { simulations. Steady-state cases will still arrive at the same answer as if this bug did not exist. }\end{array}$ \\
\hline 30 & CTF & The CTF legacy restart feature is not currently working. \\
\hline 31 & CTF & $\begin{array}{l}\text { The droplet de-entrainment model for annular mist flows that travel into top quench fronts is not } \\
\text { working correctly. The wetted perimeter that is used as input to this model is not calculated } \\
\text { correctly. This model would only be encountered in accident condition simulations (loss-of-coolant } \\
\text { accident). }\end{array}$ \\
\hline 32 & CTF & $\begin{array}{l}\text { For cases with reversed flow at the outlet, when using the outlet pressure boundary condition, and } \\
\text { with outlet voids greater than } 0.2 \text {, it is possible that the outlet void boundary condition may become } \\
\text { inconsistent and lead to the code crashing. }\end{array}$ \\
\hline 33 & CTF & $\begin{array}{l}\text { Using a inlet pressure/outlet pressure boundary condition has been observed to lead to } \\
\text { discontinuous mass flow rates in the inlet momentum cell. }\end{array}$ \\
\hline 34 & CTF & $\begin{array}{l}\text { Form losses specified for the inlet plane of the model will not be captured in the pressure losses } \\
\text { when using an inlet mass flow rate boundary condition. }\end{array}$ \\
\hline 35 & CTF & $\begin{array}{l}\text { Bundle average flow rates will always be printed as positive values, even when modeling cases with } \\
\text { reversed flow. }\end{array}$ \\
\hline 36 & CTF & $\begin{array}{l}\text { Pressure distribution is discontinuous at the inlet boundary when modeling cases with reversed } \\
\text { flow. }\end{array}$ \\
\hline 37 & CTF & $\begin{array}{l}\text { CTF predicted axial temperature distribution is nonlinear for cases with a uniform axial power } \\
\text { distribution when using a nonuniform axial mesh. }\end{array}$ \\
\hline 38 & CTF & $\begin{array}{l}\text { Pin steam rate is not correct in the HDF5 file for rods on the symmetry line when modeling } \\
\text { symmetry cases. }\end{array}$ \\
\hline
\end{tabular}




\begin{tabular}{|l|l|l|}
\hline 39 & CTF & $\begin{array}{l}\text { The CTF CHF correlation is using a statically averaged quality instead of a dynamically average } \\
\text { quality. }\end{array}$ \\
\hline 40 & CTF & $\begin{array}{l}\text { Using NC=0 (shutting off the rod conduction solution and using direct heat input to the fluid) can } \\
\text { cause numerical stability issues for some cases due to sharp changes in heat flux through the } \\
\text { transient. }\end{array}$ \\
\hline 41 & CTF & $\begin{array}{l}\text { The annular/mist interfacial drag model is not functioning correctly for cases with smooth annular } \\
\text { films. }\end{array}$ \\
\hline 42 & VERAView & $\begin{array}{l}\text { Canopy might not run on some of the most recent Linux distributions. This is based on feedback } \\
\text { from various users. }\end{array}$ \\
\hline
\end{tabular}

\section{REFERENCES}

1. Kochunas, B.M., Collins, B.S., Jabaay, D.R., Downar, T.J., and Martin, W.R, "Overview of Development and Design of MPACT: Michigan Parallel Characteristics Transport Code," International Conference on Mathematics \& Computational Methods Applied to Nuclear Science \& Engineering (M\&C 2013), Sun Valley Idaho, May 5-9, 2013.

2. Salko, R.K., and Avramova, M.N., "COBRA-TF Subchannel Thermal-Hydraulics Code (CTF) Theory Manual, Revision 0,” CASL Technical Report CASL-U-2015-0054-000 (2015).

3. “BISON References," https://bison.inl.gov/SiteAssets/bibliography.pdf (2015).

4. Godfrey, A. "VERA Core Physics Benchmark Progression Problem Specifications", CASL Technical Report CASL-U-2012-0131-004 (2014).

5. Adams, B.M., Bauman, L.E., Bohnhoff, W.J., Dalbey, K.R., Ebeida, M.S., Eddy, J.P., Eldred, M.S., Hough, P.D., Hu, K.T., Jakeman, J.D., Stephens, J.A., Swiler, L.P., Vigil, D.M., and Wildey, T.M., "Dakota, A Multilevel Parallel Object-Oriented Framework for Design Optimization, Parameter Estimation, Uncertainty Quantification, and Sensitivity Analysis: Version 6.0 User's Manual," Sandia Technical Report SAND2014-4633, July 2014. Updated November 2015 (Version 6.3).

6. Kochunas, B., Jabaay, D., Collins, B., and Downar, T., "Demonstration of Neutronics Coupled to Thermal-Hydraulics for a Full-Core Problem using COBRA-TF/MPACT", CASL Technical Report CASL-U-2014-0051-000 (2014).

7. Kochunas, B., Jabaay, D., Collins, B., and Downar, T., "Coupled Single Assembly Solution with COBRA-TF/MPACT (Problem 6)”, CASL Technical Report CASL-U-2013-0230-000 (2013). 